\title{
Voluntary Brigades Work On Integrated Fire Management in Conservation Units
}

\author{
Anderson de Freitas e Silva
}

${ }^{1}$ Brigada 1 - Minas Gerais, Brasil
E-mails to contact: defreitas.anderson@gmail.com, projetos@ brigada1.org.br

\begin{abstract}
In a current reality where environmental conservation has been related to the well-being of society as a whole and understood as an individual responsibility, voluntary work in wildfire prevention and control in protected areas is becoming more and more important. However, volunteers are not only active in prevention, control, development of new technologies, and data collection, but also disseminate a culture of protection and respect for nature which extrapolates the professional domain. Volunteer brigades involved in performing prescribed burns have significant knowledge of fire behavior and encourage civil society involvement, especially in the integrated fire management (IFM). The expertise of volunteers in preventing and fighting wildfires, coordinating teams that know the region where they operate, acting in different governance, climate realities, topographies and vegetation types can be useful tools to reduce the areas affected by high-severity fires through the IFM. Through trained volunteers, integrated fire management practices aim to minimize the costs of combat operations, stimulate research development, promote dialogue between scientific knowledge and empirical experiences, and improve the relationship between protected area managers and local communities. The prescribed burns have the potential to increase volunteer activity when compared to wildfires, as it is possible to schedule these planned actions. In addition, these activities promote high safety gains as prescribed burns tend to behave less intensely and more predictably than wildfires. The empowerment of civil society in implementing actions to conserve biodiversity in our protected areas increases the qualification of volunteer teams and reduces the vulnerability and discontinuity of activities, a situation to which government agencies are often subjected to customary budget cuts and, consequently, personnel, not always with good technical support for these tasks.
\end{abstract}

Keywords: Integrated fire management, civil society, voluntary brigade. 